Tohoku J. exp. Med., 1969, 99, 51-57

\title{
Pharmacological Studies on Reflex Renal Vasodilatation
}

\author{
Satoru Matsushita, Lie Hon Ying, Jiro Kaneko, \\ Kazuhiko Murata, Jun Fujii, Kizuku Kuramoto and Masao Ikeda \\ The Third Department of Internal Medicine (Prof. K. Nakao), \\ Faculty of Medicine, University of Tokyo, Tokyo
}

\begin{abstract}
Vasodilatation and vasoconstriction were produced in the crossperfused kidney of anesthetized dogs by intravenous injection of noradrenaline or stimulation of the cervical efferent vagus nerve. The vasomotor responses were evaluated by recording the renal blood flow with an electromagnetic flowmeter together with the systemic arterial pressure and perfusion pressure. Hydergine and $\beta$-TM10 abolished the constrictor and dilator responses, and there was no apparent tendency of specific blocking of either dilator or constrictor response in small doses. Atropine, propranolol, and hexamethonium failed to block the vasodilator response. It is concluded that the only mechanism for eliciting the renal vasodilatation is inhibition of sympathetic constrictor discharge.
\end{abstract}

There have been studies using mostly hindquarter preparation on the active and passive vasodilatation. Although reflex vasodilatation elicited by pressoreceptor mechanism had been generally considered to be passive in character, Beck and Brody ${ }^{1}$ proved that there were certain active components. There have been very few studies on the splanchnic vascular beds because of technical difficulty. In the present study, the behavior of the renal vessels to blocking agents was examined using the cross circulation of the kidney, in which reflex renal vasodilatation and vasoconstriction had been induced by pressoreceptor mechanism.

\section{Methods}

Mongrel dogs weighing 10-15 kg were used in the present investigation. Food and water were allowed ad libitum until the time of anesthesia. Anesthesia was accomplished by an intravenous injection of $60-100 \mathrm{mg}$ per $\mathrm{kg}$ of $a$-chloralose. Isolated cross perfusion of the kidney was established as described previously. ${ }^{2}$

Elevation of the recipient blood pressure was induced by intravenous injection of noradrenaline in $10 \mu \mathrm{g}$ per $\mathrm{kg}$ via the jugular vein. Hypotension was induced by electrical stimulation of the distal end of the divided cervical vagus nerve with direct current square waves of 3-5 msec in duration, at 50 impulses per second and at 2-8 volts for 10-20 seconds.

The renal blood flow decreased or increased by the baroreceptor reflex with the above procedure, while the perfusion pressure of the kidney remained unchanged. These reflex changes of the renal vascular beds having been ascertained beforehand; atropine sulphate, hexamethonium bromide, propranolol, ergot compounds (Hydergine), and $\beta$-TMI0 (SKF$6890)^{6}$ were injected into the renal artery, and the above procedure was repeated.

Received for publication, May 16, 1969. 


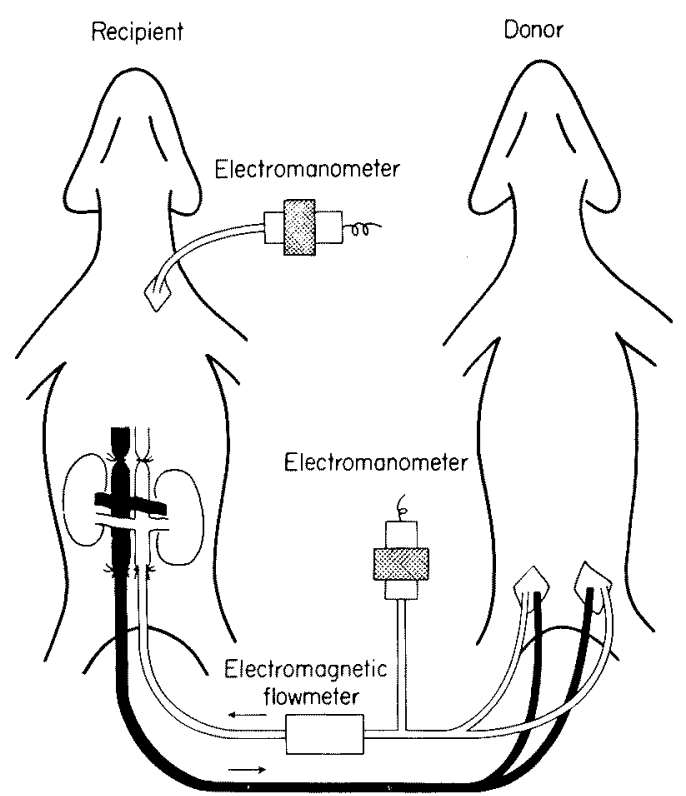

Fig. 1.

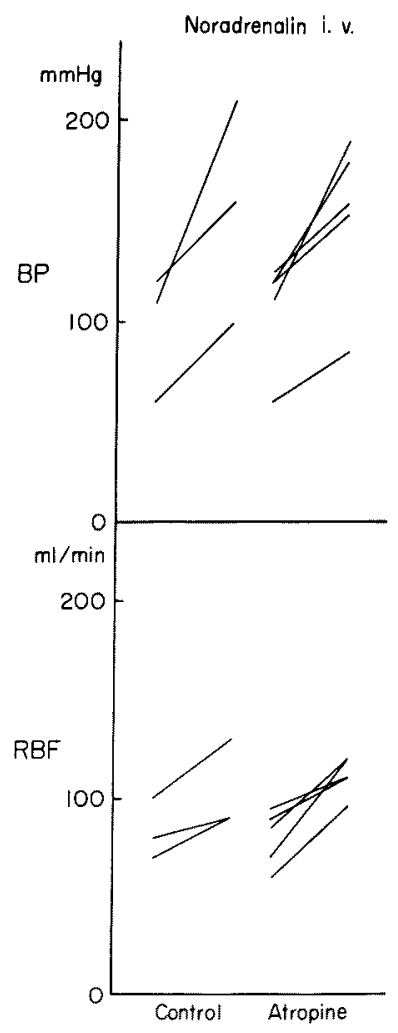

Fig. 2.

Fig. 1. Diagrammatic sketch for cross perfusion of the kidneys of a recipient $\operatorname{dog}$ by a donor.

Fig. 2. Elevation of recipient blood pressure (BP) by intravenous noradrenaline and reflex increase of renal blood flow (RBF) before and after intra-renal-arterial atropine, showing no remarkable change in reflex renal vasodilatation.

\section{Results}

1) Atropine sulphate in $0.25 \mathrm{mg}$ per kidney did not block the noradrenalineinduced reflex vasodilatation of the kidneys in three recipient dogs (Fig. 2).

2) Hexamethonium bromide in $1.2-5 \mathrm{mg}$ per kidney also failed to inhibit the reflex vasoconstriction and vasodilatation induced by the vagus stimulation and by noradrenaline, respectively, in three recipients (Fig. 3).

3) Hydergine (a mixture of equal methanesulfonates of dihydroergocornine, of dihydroergocrystine, and of dihydroergokryptine) in $0.15 \mathrm{mg}$ per kidney, sufficient to block intra-arterially injected noradrenaline, abolished both reflex vasodilatation and vasoconstriction in six recipients (Fig. 4). Small doses of 0.005-0.05 mg per kidney blocked the reflex less completely. However, there was no specific inhibition of vasodilatation.

${ }^{*} \beta-$ TM10 was obtained through the courtesy of Smith Kline and French Overseas Company. 


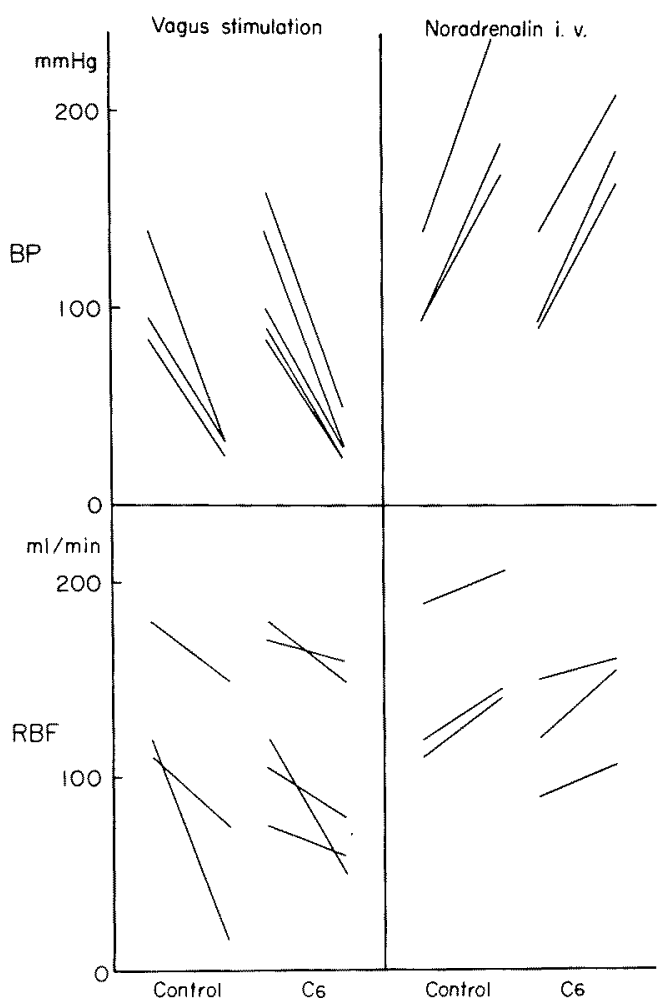

Fig. 3. Hypotension induced by the electrical stimulation of cervical efferent vagus nerve and hypertension by intravenous noradrenaline causing reflex decrease and increase of renal blood flow. Pretreatment with intra-renal-arterial hexamethonium (C6) does not affect the reflex renal vascular change.

4) Propranolol in $0.005-2.5 \mathrm{mg}$ per kidney was sufficient to block isoproterenol, but abolished neither the reflex renal vasodilatation nor vasoconstriction in five recipients, when injected intra-arterially (Fig. 5).

5) $\beta$-TM10 in $15-30 \mathrm{mg}$ per kidney abolished both reflex vasodilatation and vasoconstriction in four recipients. With smaller doses of 1-5 mg per kidney, the reflex was inhibited incompletely. However, specific blockade of vasoconstriction was not demonstrated (Figs. 6 and 7 ).

\section{Discussion}

The above results indicate that the renal vessels are innervated by the adrenergic constrictor sympathetic nerve, and the reflex dilatation of the renal vessels is induced passively by vascular relaxation due to interruption of tonic release of a constrictor substance. Adrenergic sympathetic nerve is not ganglionated within the kidney. In our previous papers ${ }^{2,3}$ it was shown that renal vascular reflex changes are mediated via the splanchnic nerves, and there are 


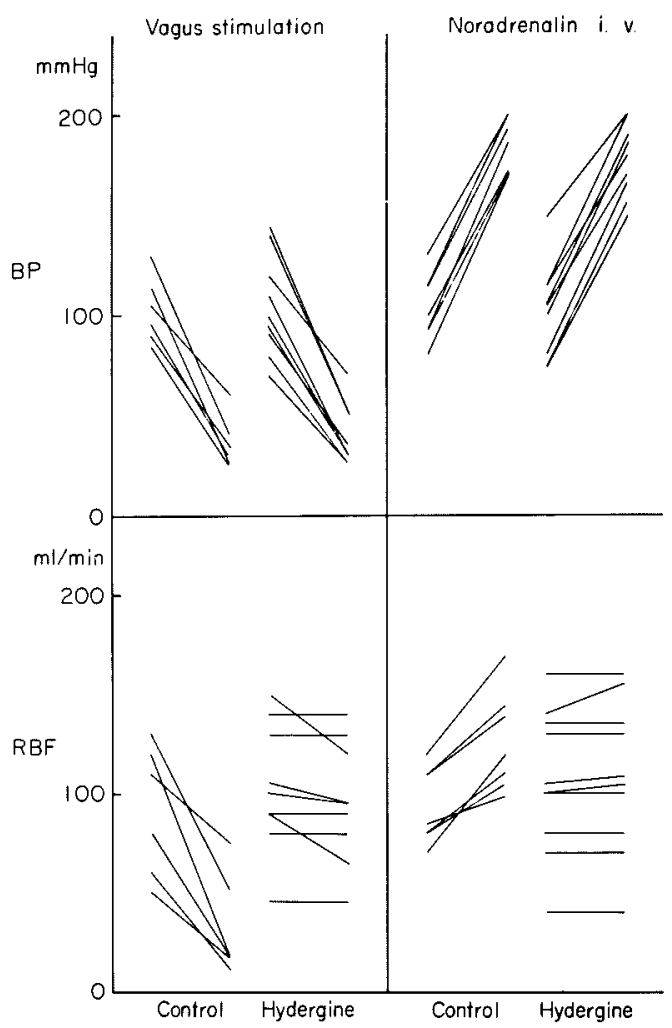

Fig. 4. The effect of Hydergine on reflex renal vasomotor change showing incomplete or complete inhibition.

no vagal control on renal circulation. Since atropine fails to block the renal reflex vasodilatation, the sympathetic cholinergic system, ${ }^{4}$ even if present in renal vessels, does not participate in the baroreceptor reflex vasodilatation.

According to Sakuma and Beck, ${ }^{5}$ optimum doses of $\beta$-TM10, which is adrenergic antirelease agent like TM10, bretylium or guanethidine, dissociate active and passive vasodilatation. In their experiments using innervated hindquarter perfused with a pump, reflex dilatation remained after abolition of sympathetic tone by optimal doses of $\beta$-TM10. The result demonstrated that active reflex dilatation could occur in the absence of sympathetic tone. By the use of amine oxidase inhibitors and $\beta$-TM10, they further demonstrated the presence of a large active component of reflex dilatation when considerable sympathetic tone existed or when the potential for passive dilatation was large. ${ }^{6}$ However, our results on the renal vessels are not in accord with theirs. Reflex constriction was blocked just as reflex dilatation with small doses of $\beta$-TM10. The ergot compounds also eliminated the active component of reflex dilatation in small doses without producing adrenergic blockade in hind limb preparation ${ }^{1}$ but did not show the same effects. One of the reasons for the active vasodilatation ${ }^{5}$ was that reflex dilatation induced by intravenous adrenaline could exceed the vasodilatation after sympathectomy or 


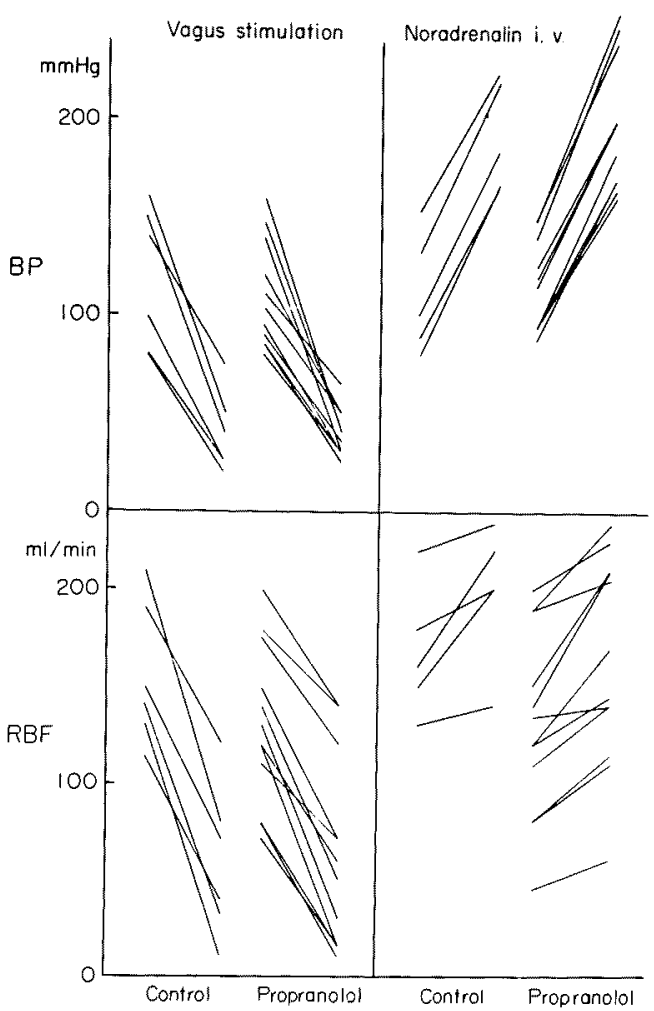

Fig. 5. The effect of propranolol on reflex renal vasomotor change showing no remarkable effect.

ganglion blockade. In our series of experiments, the sympathectomy was not performed. However, from the experiments on the effect of splanchnicectomy, ${ }^{2}$ it is our impression that the renal reflex dilatation would not exceed the sympathectomy-induced vasodilatation.

In our experiments the vasomotor response was evaluated by recording the blood flow with constant perfusion pressure. This is different from the Beck's experiment on the hind limb preparation in which the vasomotor response was evaluated by recording the perfusion pressure with constant blood flow using a pump. The former might be more sensitive to the change of capacitance vessels, whereas the latter might be so to the change of resistance vessels. Despite this difference in the methods of detecting the vasomotor response, it is still likely that the renal vascular neurogenic control is different from one of the skeletal muscle in respect to reflex vasodilatation. Although both the renal and skeletal muscle vascular beds have a high basal tone and also a prominent autoregulation, the potencies of probable receptors in arterioles of both organs are quite different.? 


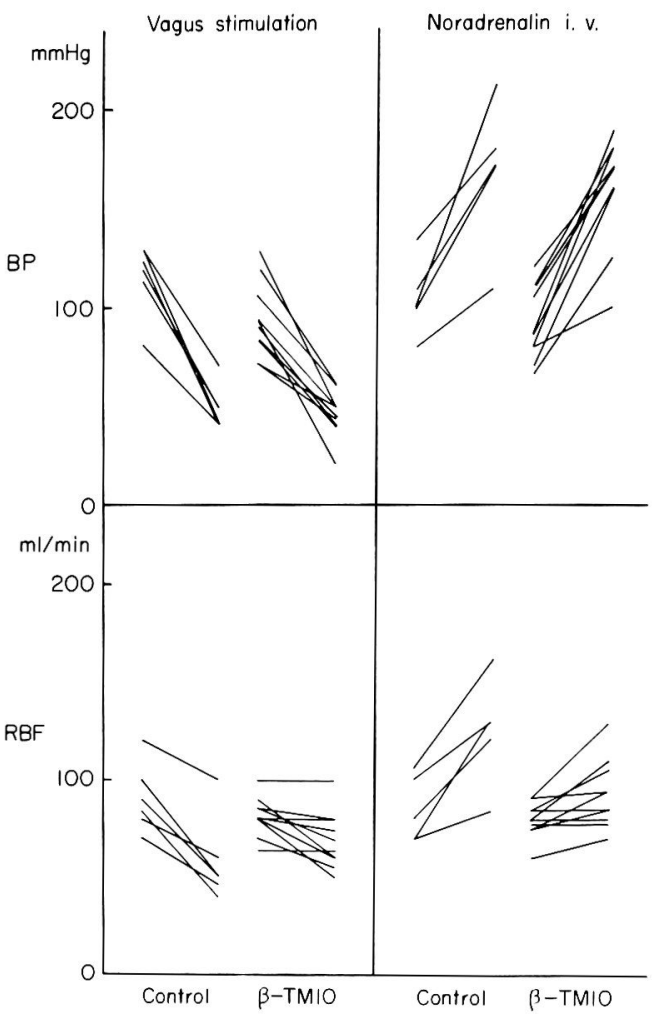

Fig. 6. The effect of $\beta$-TM10 (SKF-6890A) on reflex renal vasomotor change showing complete or incomplete inhibition.

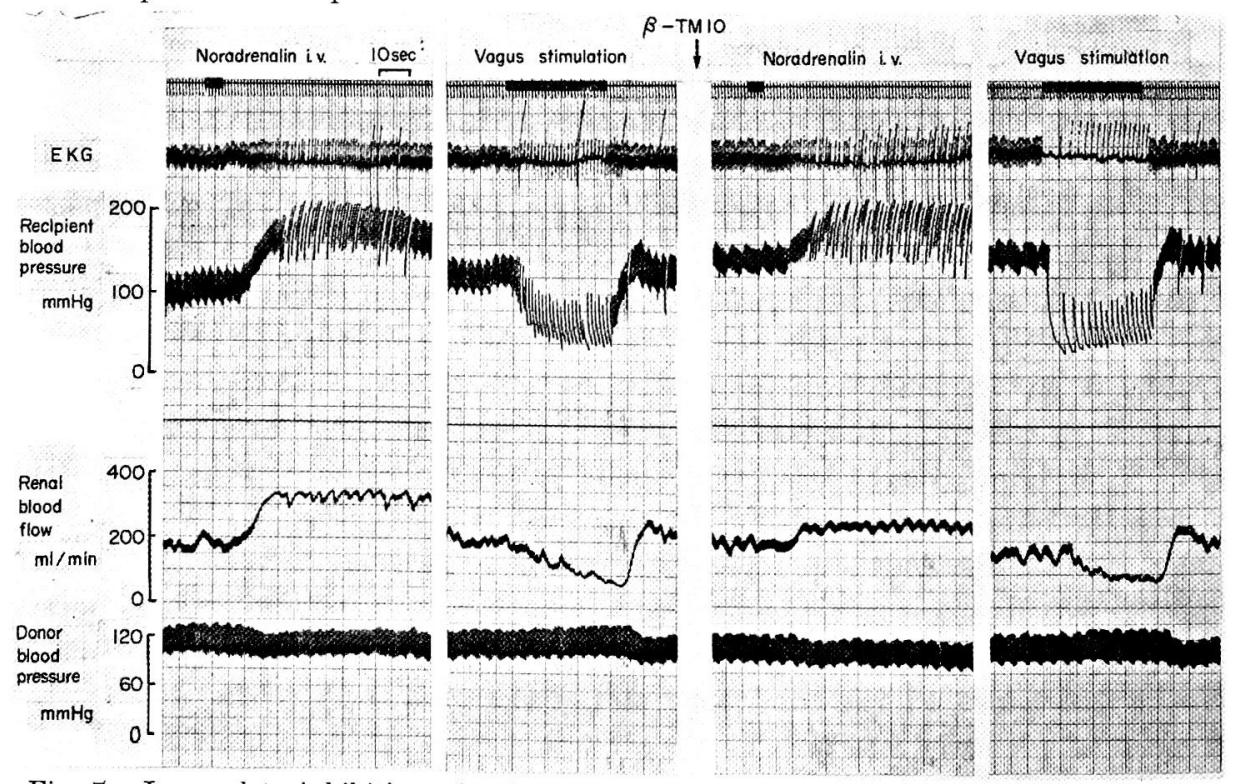

Fig. 7. Incomplete inhibition of reflex renal vasomotor response by $10 \mathrm{mg}$ of $\beta-\mathrm{TM} 10$ injected intra-renal-arterially. Both the constrictor and dilator responses are inhibited. Later, with additional doses the reflex was completely abolished. 


\section{References}

1) Beck, L. \& Brody, M.J. The physiology of vasodilation. Angiology, 1961, 12, 202222.

2) Matsushita, S. Reflex control of the renal circulation. Tohoku J.exp. Med., 1967, 9, $261-270$.

3) Matsushita, S. Absence of vagal control on renal circulation. Tohoku J. exp. Med., 1967, 91, 271-275.

4) Frumin, M.J., Ngai, S.H. and Wang, S.C. Evaluation of vasodilator mechanisms in the canine hind leg; question of dorsal root participation. Amer. J. Physiol., 1953, $173,428-436$.

5) Sakuma, A. \& Beck, L. Pharmacological evidence for active reflex dilatation. Amer. J. Physiol., 1961, 201, 129-133.

6) Beck, L. Active reflex dilatation in the innervated perfused hind limb of the dog. Amer. J. Physiol., 1961, 201, 123-128.

7) Green, H.D. \& Kepchar, J.H. Control of peripheral resistance in major systemic vascular beds. Physiol. Rev., 1959, 39, 617-686. 